\begin{tabular}{|c|c|c|c|}
\hline 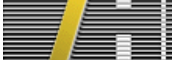 & 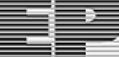 & PuBlisheD & FOR SISSA BY $\underline{\underline{y}}$ SPRINGER \\
\hline & & & $\begin{array}{l}\text { RECEIVED: April 26, } 2016 \\
\text { ACCEPTED: May 9, } 2016 \\
\text { PUBlished: May 18, } 2016\end{array}$ \\
\hline
\end{tabular}

\title{
A model for the LHC diboson excess
}

\author{
Manuel Buen-Abad, Andrew G. Cohen and Martin Schmaltz \\ Physics Department, Boston University, \\ Boston, MA 02215, U.S.A. \\ E-mail: buenabad@bu.edu, cohen@bu.edu, schmaltz@bu.edu
}

ABSTRACT: The first run of the LHC showed hints of a new resonance with mass near $1.9 \mathrm{TeV}$ decaying into electroweak gauge boson pairs as well as into dijets. While Run 2 has neither confirmed nor ruled out such a resonance, it has yielded new constraints on models attempting to explain these decays. Additionally in $W^{\prime}$ models where this new resonance is a charged vector boson that is a weak isospin singlet there is the potential for conflict with the electroweak precision $T$ parameter. We construct variants of a $W^{\prime}$ resonance model that provide an excellent fit to both Run 1 and Run 2 data, as well as electroweak precision measurements. The model also predicts a neutral vector boson, a $Z^{\prime}$, with mass close to $3 \mathrm{TeV}$. This $Z^{\prime}$ is compatible with the intriguing Run 2 observation of a dielectron pair with invariant mass of $2.9 \mathrm{TeV}$ at CMS.

Keywords: Beyond Standard Model, Gauge Symmetry

ARXiv EPrint: 1604.03578 


\section{Contents}

1 Introduction 1

$\begin{array}{lll}2 & \text { Symmetry breaking } & 7\end{array}$

3 Fermion masses and mixings $\quad 8$

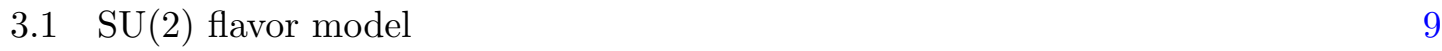

$\begin{array}{lll}3.2 & \mathrm{SU}(3) \text { flavor model } & 11\end{array}$

4 Fit to precision electroweak and LHC data $\quad 11$

$\begin{array}{lll}4.1 & \text { Models with triplet breaking } k=\sqrt{2} & 15\end{array}$

$\begin{array}{ll}4.2 \text { Models with doublet breaking } k=1 & 16\end{array}$

\section{Introduction}

Several results from ATLAS [1-5] and CMS [6-11] in Run 1 of the LHC hint at the existence of a narrow resonance with decays to dijet and diboson final states and a mass near $2 \mathrm{TeV}$. While none of the individual deviations from the Standard Model (SM) have more than $3 \sigma$ significance, the fact that several different searches find excesses which can be explained with a single bosonic resonance is intriguing $[12,13]$. There are many interesting aspects of this data, but the feature that we will focus on is the apparent decay of the massive resonance to electroweak ( $\mathrm{EW}$ ) gauge boson pairs.

A particularly attractive interpretation of the Run 1 data is a new massive charged gauge boson, a $W^{\prime}$, with a mass close to $1.9 \mathrm{TeV}$ [14-36]. A combination of the Run 1 ATLAS and CMS data obtains a good fit with a $W^{\prime}$ mass close to $1.9 \mathrm{TeV}$ and a $W^{\prime} \rightarrow W Z$ cross section of $5.3_{-2.0}^{+2.3} \mathrm{fb}$ [12]. Run 1 also showed evidence for a dijet decay mode $W^{\prime} \rightarrow j j$ with a cross section on the order of $50 \mathrm{fb}$, with significant uncertainty [13].

Toward the end of 2015 the first results from Run 2 of the LHC at $13 \mathrm{TeV}$ were announced. For most channels relating to the diboson excess the Run 2 sensitivity was somewhat below that of Run 1, and these new results neither confirm nor exclude the signal. A combination of the most sensitive channels in ATLAS [37-40] and CMS [41] from Run 2 yields a $95 \%$ exclusion bound on the $W^{\prime} \rightarrow W Z$ rate at $13 \mathrm{TeV}$ for a $1.9 \mathrm{TeV} W^{\prime}$ of $25 \mathrm{fb}$. In addition a Run 2 ATLAS analysis places a $95 \%$ confidence upper limit on the dijet rate of about $150 \mathrm{fb}$ [42]. The parton luminosities appropriate for $W^{\prime}$ production are approximately 6 times larger at $13 \mathrm{TeV}$ compared to $8 \mathrm{TeV}$, and thus these Run 2 limits correspond to $95 \%$ confidence Run 1 limits of $\sigma_{W Z}<4 \mathrm{fb}$ and $\sigma_{j j}<25 \mathrm{fb}$.

A charged $W^{\prime}$ gauge boson of this sort must arise from a non-abelian group including $\mathrm{SU}(2)$ and therefore comes with a neutral partner, a $Z^{\prime}$. We imagine an effective theory below some scale $f \gg 246 \mathrm{GeV}$ in which the unbroken SM gauge group $\mathrm{SU}(2)_{\mathrm{SM}} \times \mathrm{U}(1)_{Y}$ 
is supplemented by the new massive gauge bosons, one or more Higgs doublets, and where all operators of dimension greater than four are suppressed by the high scale. To produce the diboson signal the $W^{\prime}$ should decay to pairs of EW gauge bosons. This allows two possibilities for the quantum numbers of $V^{\prime}=W^{\prime}, Z^{\prime}$ under the SM gauge group $\mathrm{SU}(2)_{\mathrm{SM}} \times \mathrm{U}(1)_{Y}:$

- $V^{\prime}$ is a triplet under $\mathrm{SU}(2)_{\mathrm{SM}}$ and has zero hypercharge. We refer to the resulting massive vector bosons as "left-handed" and this model as the "left-handed" model.

- $V^{\prime}$ is a singlet under $\mathrm{SU}(2)_{\mathrm{SM}}$ and the hypercharges are $\pm 1,0$. We refer to the resulting massive vector bosons as "right-handed" and this model as the "right-handed" model. This case is the focus of this work.

There are no renormalizable, gauge-invariant operators in this effective theory that couple the $V^{\prime}$ to pairs of SM gauge bosons and produce the diboson signal. We can obtain the desired decay of the massive resonances through higher dimension operators, but these are generically too small. Alternatively the massive resonance may couple to the longitudinal components of the $W$ and $Z$ after EW symmetry breaking. That is, the scalar fields that acquire EW vevs (and contain the longitudinal components of the $W$ and the $Z$ ) can couple at dimension four to the massive resonances. In our effective theory the only relevant operators take the form of the massive gauge bosons times currents constructed from the Higgs fields. For the left-handed case these currents must be $\mathrm{SU}(2)_{\mathrm{SM}}$ triplets and $\mathrm{U}(1)_{Y}$ singlets, while for the right-handed case they must by $\mathrm{SU}(2)_{\mathrm{SM}}$ singlets with hypercharge $\pm 1,0$. We denote them generically as

$$
g_{V^{\prime}} V_{\mu}^{\prime} \Phi i D^{\mu} \Phi
$$

where $g_{V^{\prime}}$ is a coupling constant, $D$ is the covariant derivative including the electroweak gauge fields, $\Phi$ is a Higgs field (or its conjugate) and we have suppressed explicit indices. The form of these operators is one of the reasons that a heavy vector resonance with a diboson decay mode is of such interest: this decay is a direct measurement of EW symmetry breaking and probes the details of the Higgs vevs.

In addition to providing the diboson decay, the operator (1.1) includes mass mixing of the heavy resonances with the $W$ and the $Z$. This mixing may shift the mass of the $W$ relative to the $Z$. While this shift is small, the extraordinarily precise measured values of these masses significantly constrain such an effect: electroweak precision measurements preclude a large correction to the $T$ parameter. There is a straightforward way to help protect against such a correction: incorporate a custodial $\mathrm{SU}(2)$ symmetry. This is automatic in the left-handed model where the heavy resonances are a triplet under $\mathrm{SU}(2)_{\mathrm{SM}}$. The right-handed model has no such protection: the $W^{\prime}$ and $Z^{\prime}$ are not members of an $\mathrm{SU}(2)_{\mathrm{SM}}$ triplet and their mixing with the $W$ and $Z$ violates custodial $\mathrm{SU}(2)$. Therefore the operators responsible for the heavy vector decay into dibosons may also generate a non-zero value of the $T$ parameter. This is the main topic of our paper: exploration of the tension between the constraints on the $T$ parameter and the diboson branching fraction for 
models with a right-handed $W^{\prime}$. We will find that relaxing this tension prefers a $Z^{\prime}$ mass right around $3 \mathrm{TeV}$.

Upon substituting EW symmetry breaking vevs and allowing for independent couplings of the heavy $W^{\prime}$ and $Z^{\prime}$ resonances the operators of (1.1) correspond to the mixing terms

$$
\kappa_{W} M_{W}^{2} W^{\prime-} W^{+}+\text {h.c. }+\kappa_{Z} M_{Z}^{2} \cos \theta_{W} Z^{\prime} Z
$$

where we have parameterized the couplings relative to the electroweak gauge boson masses and the electroweak mixing angle $\left(\cos \theta_{W} \equiv M_{W} / M_{Z}\right)$ for convenience.

The coupling $\kappa_{W}$ determines the rate for $W^{\prime}$ decay to $W Z$

$$
\Gamma\left(W^{\prime} \rightarrow W Z\right)=\left|\kappa_{W}\right|^{2} \frac{g^{2}}{192 \pi} M_{W^{\prime}}
$$

where $g$ is the $\mathrm{SU}(2)_{\mathrm{SM}}$ gauge coupling. The corresponding rate measured at the LHC is the product of the $W^{\prime}$ production rate times the branching fraction of the $W^{\prime}$ into $W Z$. Significant production of the $W^{\prime}$ requires a coupling $g_{u d}$ to the first family of quarks. In a straightforward implementation of an $\mathrm{SU}(2)_{M}$ gauge theory in which the quarks are doublets under $\mathrm{SU}(2)_{M}[17,20,25,30,43], g_{u d}$ is simply the gauge coupling $g_{M}$. The $W^{\prime}$ then couples universally to all three families of quarks, with a decay rate

$$
\Gamma\left(W^{\prime} \rightarrow q \bar{q}\right)=3 \frac{g_{M}^{2}}{16 \pi} M_{W^{\prime}}
$$

However the rate for $W^{\prime} \rightarrow W Z$ is determined by the same gauge coupling times a factor for the fraction of the longitudinal $W$ and $Z$ bosons contained in the scalar field $\Phi$. This fraction is necessarily less than one, and therefore the $W Z$ decay rate is bounded by $\Gamma\left(W^{\prime} \rightarrow W Z\right) \leq M_{W^{\prime}} g_{M}^{2} /(192 \pi)$. This leads to a lower bound on the dijet rate relative to the $W Z$ event rate:

$$
\sigma_{j j} \geq 36 \sigma_{W Z}
$$

A Run $1 W Z$ signal of a few femtobarns thus requires a Run 1 dijet rate in excess of a hundred femtobarns. Such a large dijet rate is fully excluded by the Run 2 data. For this reason the models we construct will incorporate fermion mixing, allowing the $W^{\prime}$ coupling to first family quarks $g_{u d}$ to differ from the gauge coupling $g_{M}$. Mixing of fermions inevitably involves issues of flavor, and without fine tuning or additional flavor symmetries we run the risk of significant flavor changing neutral currents. We therefore include flavor symmetry to afford some protection against these dangerous effects. We will consider two examples: one in which the coupling to all three families is universal; and another with universal couplings to first and second families but no coupling of the $W^{\prime}$ to the third.

It is convenient to write the $W Z$ branching fraction in terms of the branching fraction of the $W^{\prime}$ to quarks. Defining $B_{j j} \equiv \mathrm{B}\left(W^{\prime} \rightarrow q \bar{q}\right)$ this is $\mathrm{B}\left(W^{\prime} \rightarrow W Z\right)=B_{j j} \cdot \Gamma\left(W^{\prime} \rightarrow\right.$ $W Z) / \Gamma\left(W^{\prime} \rightarrow q \bar{q}\right) .{ }^{1}$ With $N_{f}$ the number of families that the $W^{\prime}$ couples to (either 2 or 3 )

\footnotetext{
${ }^{1}$ An additional diboson signal stems from the $W^{\prime}$ decay to Higgs particles, $W^{\prime} \rightarrow W h$. In models with a single Higgs doublet the rate for this mode is equal to that of $W^{\prime} \rightarrow W Z$. With multiple Higgs doublets the rates may differ. However since the observed Higgs particle has couplings consistent with the full vev of $246 \mathrm{GeV}$ this suggests that the observed Higgs couples to $W^{\prime}$ like the full vev as well. In this case the $W^{\prime} \rightarrow W h$ rate is again the same as the $W^{\prime} \rightarrow W Z$ rate.
} 
the decay rate to quarks is

$$
\Gamma\left(W^{\prime} \rightarrow q \bar{q}\right)=N_{f} \frac{g_{u d}^{2}}{16 \pi} M_{W^{\prime}}
$$

The rate for $W^{\prime}$ production may be computed by integrating the production cross section over parton distribution functions

$$
\sigma\left(p p \rightarrow W^{\prime}\right)=\frac{\pi}{6} \frac{g_{u d}^{2}}{s} \int_{M_{W^{\prime}}^{2} / s}^{1} \frac{d x}{x}\left[f_{u}(x) f_{\bar{d}}\left(\frac{M_{W^{\prime}}^{2}}{x s}\right)+f_{d}(x) f_{\bar{u}}\left(\frac{M_{W^{\prime}}^{2}}{x s}\right)\right] \simeq g_{u d}^{2} 0.8 \mathrm{pb} .
$$

Here $\sqrt{s}=8 \mathrm{TeV}$ is the collider center of mass energy, the $f_{i}(x)$ are the parton distribution functions, and we have summed over both first and second family quarks. For our numerical results we use MSTW parton distribution functions [44] with NLO K-factors taken from [45-47]. The diboson cross section from $W^{\prime}$ production $\sigma_{W Z}$ is then the product

$$
\begin{aligned}
\sigma_{W Z}=\sigma\left(p p \rightarrow W^{\prime}\right) \mathrm{B}\left(W^{\prime} \rightarrow W Z\right) & = \\
\sigma\left(p p \rightarrow W^{\prime}\right) B_{j j} \frac{\Gamma\left(W^{\prime} \rightarrow W Z\right)}{\Gamma\left(W^{\prime} \rightarrow q \bar{q}\right)} & = \\
\left|\kappa_{W}\right|^{2} & \frac{B_{j j}}{N_{f}} \frac{g^{2}}{12} 0.8 \mathrm{pb}=\left|\kappa_{W}\right|^{2} \frac{B_{j j}}{N_{f}} 28 \mathrm{fb} .
\end{aligned}
$$

Note that the dependence on the fermion coupling to the $W^{\prime}$ has been subsumed in the branching fraction to quarks. We then have a prediction for $\sigma_{W Z}$ with $\kappa_{W}$ and $B_{j j} / N_{f}$ as the only free parameters. As a rough benchmark, a signal of $4 \mathrm{fb}$ with $N_{f}=2$ corresponds to $B_{j j}\left|\kappa_{W}\right|^{2} \simeq .29$. Since $B_{j j}<1$ this means that $\kappa_{W} \gtrsim .5$ to obtain this cross section.

We may develop some intuition for the precision electroweak constraints that apply to (1.2) by noting that the most precisely measured electroweak parameters are the Fermi constant $G_{F}$, the fine structure constant at the $Z$ mass $\alpha\left(M_{Z}\right)$, the mass of the $Z, M_{Z}$, and the mass of the $W, M_{W}$. In the SM any three of these observables may be used to fix the parameters in the gauge sector of the theory $\left(g, g^{\prime}, v\right)$ and then one prediction for the remaining parameter may be obtained. ${ }^{2}$ The same procedure may be applied including the operators of (1.2) where now the prediction depends on the parameters $\kappa_{W, Z}$. It is convenient to phrase this prediction as $M_{W}^{2} / M_{W 0}^{2}-M_{Z}^{2} / M_{Z 0}^{2}$ where the subscript 0 indicates the SM value. The SM prediction for this parameter is clearly zero, whereas in the resonance model we need only compute shifts in masses from SM values: $\delta M_{W}^{2} / M_{W 0}^{2}-\delta M_{Z}^{2} / M_{Z 0}^{2}$. In the absence of couplings of the heavy resonances other than those in (1.2) this is just the conventionally defined $T$ parameter [48] given by (to leading order in inverse powers of the heavy masses)

$$
\alpha\left(M_{Z}\right) T=-\left|\kappa_{W}\right|^{2} \frac{M_{W}^{2}}{M_{W^{\prime}}^{2}}+\kappa_{Z}^{2} \cos ^{2} \theta_{W} \frac{M_{Z}^{2}}{M_{Z^{\prime}}^{2}}=\frac{M_{W}^{2}}{M_{W^{\prime}}^{2}}\left\{-\left|\kappa_{W}\right|^{2}+\kappa_{Z}^{2} \frac{M_{W^{\prime}}^{2}}{M_{Z^{\prime}}^{2}}\right\}
$$

There are no tree level contributions to the other prominent electroweak precision parameter $S$. Precision measurements constrain $\alpha\left(M_{Z}\right) T$ to be less than $10^{-3}$ which implies $\kappa_{W, Z}$

\footnotetext{
${ }^{2}$ This prediction is only weakly dependent on the other parameters of the model, such as $\alpha_{s}$, the Higgs mass and quartic coupling, and the quark Yukawa couplings.
} 


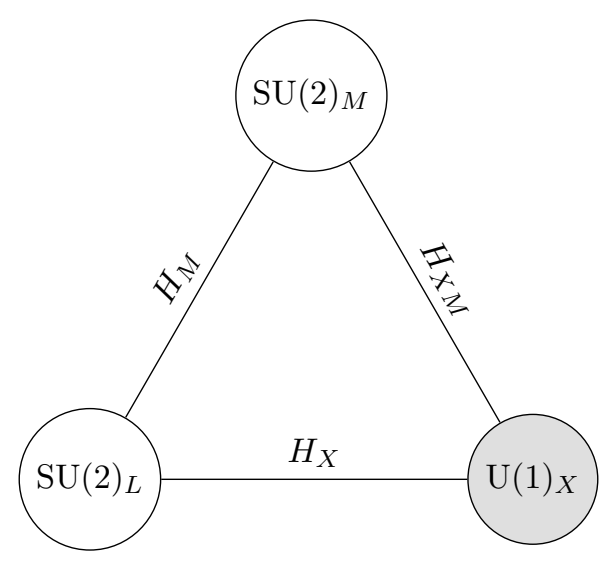

Figure 1. Theory space diagram representing the bosonic field content for the models described in the text.

no larger than of order one, or cancellations between the $W^{\prime}$ and $Z^{\prime}$ contributions. With $S=0$ the precision fit has a preference for positive values of $T, \alpha T=(4 \pm 2.4) \times 10^{-4}$, suggesting that the $Z^{\prime}$ contribution should be larger than that of the $W^{\prime}$.

The inherent tension between the diboson signal and the $T$ parameter is already evident in these general expressions. A large diboson rate requires a large value for $\kappa_{W}\left(B_{j j}\right.$ can only suppress the rate), but this pushes the $T$ parameter in the wrong direction. This can be compensated by a contribution from the $Z^{\prime}$ through $\kappa_{Z}$, but only if the $Z^{\prime}$ is not too heavy.

To refine this constraint on the $Z^{\prime}$ mass we need to make some choices. We may construct a right-handed $W^{\prime}$ model starting with the gauge group $\mathrm{SU}(2)_{L} \times \mathrm{SU}(2)_{M} \times \mathrm{U}(1)_{X}$ and breaking $\mathrm{SU}(2)_{M} \times \mathrm{U}(1)_{X} \rightarrow \mathrm{U}(1)_{Y}$ at the scale $f$. The resulting massive gauge bosons are the right-handed $W^{\prime}$ and $Z^{\prime}$ and below this scale we have the desired effective theory. Including a $(1, R)_{(R-1) / 2}$ field $H_{X M}$ (for some non-trivial representation of dimension $R$ ) that acquires a large vev $f / \sqrt{2}$ accomplishes the desired breaking. The hypercharge gauge coupling is $g^{\prime}=g_{M} g_{X} / \sqrt{g_{M}^{2}+g_{X}^{2}} \equiv g_{M} \sin \theta_{M}$. The smallest such representation is $R=$ 2 , the doublet. However as we will see this leads to either a poor precision fit or fine tuning. Therefore we prefer an $\mathrm{SU}(2)_{M}$ triplet, $R=3$. In this case the $Z^{\prime}$ mass is $M_{Z^{\prime}}=\sqrt{2} M_{W^{\prime}} / \cos \theta_{M}$.

We must also include scalar field representations that contain Higgs doublets following this breaking. These are representations of the form $(2, R)_{X}$. The smallest such representations, each containing four real fields, are a complex doublet $(2,1)_{1 / 2}$ field $H_{X}$ and a real "bi-doublet" $(2,2)_{0}$ field $H_{M} \cdot{ }^{3}$ The model including these representations is nicely summarized by the theory space diagram of figure 1 .

\footnotetext{
${ }^{3}$ Since $\mathrm{SU}(2) \times \mathrm{SU}(2) \sim \mathrm{SO}(4)$ the real bidoublet may be equivalently thought of as the vector of $\mathrm{SO}(4)$. We may represent this field in a variety of ways: as a complex two component vector $\left(\begin{array}{c}\phi^{+} \\ \phi^{0}\end{array}\right)$ (as we choose here); as a $2 \times 2$ matrix $\left(\begin{array}{c}\phi^{0 *} \\ \phi_{\phi^{+*}} \phi_{\phi^{0}}\end{array}\right)$; or as a 4-component column vector formed from the real and imaginary parts of $\phi^{0}$ and $\phi^{+}$.
} 
The mixing operators in (1.1) then take the form

$$
\begin{aligned}
& \frac{g_{M}}{2} \sqrt{2} W_{\mu}^{\prime-} H_{M}^{T} i \sigma^{2} i D^{\mu} H_{M}+\text { h.c. } \\
& \quad+\frac{g_{M}}{2} \cos \theta_{M} Z_{\mu}^{\prime} H_{M}^{\dagger} i D^{\mu} H_{M}-\frac{g_{X}}{2} \sin \theta_{M} Z_{\mu}^{\prime} H_{X}^{\dagger} i D^{\mu} H_{X}
\end{aligned}
$$

Our models will include two of the bidoublets $H_{M}$ and, for the moment, we will ignore any vevs for the $H_{X}$ fields. Each of these bidoublets is an SM Higgs field and we therefore have a multi-Higgs doublet model.

The sum $\sum\left|v_{M}\right|^{2}+\left|v_{X}\right|^{2} \equiv v^{2}$ is constrained to the electroweak value $v^{2}=(246 \mathrm{GeV})^{2}$. Note that the phases in the vevs of these bidoublets are not necessarily aligned and $t^{2} \equiv$ $\left|\sum v_{M}^{2}\right| / v^{2} \leq 1$. The $W^{\prime}$ mixing in (1.10) is sensitive to these phases and thus $t$ appears in $\kappa_{W}$. Both $\kappa_{W}$ and $\kappa_{Z}$ are readily computed (ignoring $v_{X}$ )

$$
\begin{aligned}
& \kappa_{W}=\frac{g_{M}}{g} t^{2}=\frac{\tan \theta_{W}}{\sin \theta_{M}} t^{2} \\
& \kappa_{Z}=\frac{g_{M}}{g} \cos \theta_{M}=\frac{\tan \theta_{W}}{\sin \theta_{M}} \cos \theta_{M}
\end{aligned}
$$

so that (1.9) gives

$$
\alpha\left(M_{Z}\right) T=\frac{\tan ^{2} \theta_{W}}{\sin ^{2} \theta_{M}} \frac{M_{W}^{2}}{M_{W^{\prime}}^{2}}\left[\frac{\cos ^{4} \theta_{M}}{2}-t^{4}\right]
$$

When the phases of the Higgs fields are all aligned (or in the case of a single bidoublet field in which case the phase is necessarily aligned) $t=1$ and (1.12) gives

$$
\alpha\left(M_{Z}\right) T=-\tan ^{2} \theta_{W} \frac{M_{W}^{2}}{M_{W^{\prime}}^{2}}\left(\frac{2-\cos ^{4} \theta_{M}}{2 \sin ^{2} \theta_{M}}\right) \lesssim-5.3 \times 10^{-4}
$$

where we have set $M_{W^{\prime}}=1.9 \mathrm{TeV}$. Although the $T$ parameter in (1.13) is not much larger than the experimental uncertainty, it is unfortunately negative, and more than 3 sigma away from the experimental value. The negative definiteness of the result reflects the dominance of the $W^{\prime}$ contribution over that of the $Z^{\prime}$ for all values of the $Z^{\prime}$ mass. Evading the constraint in (1.13) is necessary for a good precision fit, and (1.12) demonstrates that this requires non-aligned vevs so that $t^{2}<1$.

With these ingredients in place, (1.8) and (1.12) relate $\alpha T, \sigma_{W Z}, B_{j j} / N_{f}$ and the $Z^{\prime}$ mass:

$$
2 \frac{M_{W^{\prime}}^{4}}{M_{Z^{\prime}}^{4}} \frac{1}{1-2 M_{W^{\prime}}^{2} / M_{Z^{\prime}}^{2}}=\alpha\left(M_{Z}\right) T \frac{1}{\tan ^{2} \theta_{W}} \frac{M_{W^{\prime}}^{2}}{M_{W}^{2}}+\frac{N_{f}}{B_{j j}} \frac{\sigma_{W Z}}{28 \mathrm{fb}}
$$

We may trade $B_{j j}$ for the dijet resonance cross section at $1.9 \mathrm{TeV}$. Assuming all decays aside from dijets and dibosons are small

$$
B_{j j}=\frac{\sigma_{j j}}{\sigma_{j j}+2 \sigma_{W Z}}
$$

where we have used $\sigma_{W Z}+\sigma_{W h} \simeq 2 \sigma_{W Z}$.

In fact $M_{Z^{\prime}}$ as determined by (1.14) and (1.15) is rather insensitive to the values of the dijet cross section preferred by the data. For $\sigma_{j j}$ much larger than $\sigma_{W Z} \sim 4 \mathrm{fb}$ the 
dijet branching fraction $B_{j j} \simeq 1$ and any dependence on $\sigma_{j j}$ disappears. Smaller values of $\sigma_{j j}$ suppress $B_{j j}$ and require larger values of $\kappa_{W}$, in turn requiring a smaller $Z^{\prime}$ mass to fit the $T$ parameter. Note that for very small values of the dijet cross section, $\sigma_{j j} \ll 1 \mathrm{fb}$, the last term in (1.14) grows large, and avoiding unacceptably large corrections to the $T$ parameter requires fine tuning of the $Z^{\prime}$ mass such that the left hand side of this equation compensates. To avoid this tuning we will prefer parameters which yield a dijet cross section greater than a few femtobarns.

Fixing $M_{W^{\prime}}=1.9 \mathrm{TeV}, \sigma_{W Z}=4 \mathrm{fb}, \sigma_{j j}>1 \mathrm{fb}$, and allowing $\alpha T$ to vary over its $1 \sigma$ range we find a range for the $Z^{\prime}$ mass of

$$
2.8 \mathrm{TeV}<M_{Z^{\prime}}<3.2 \mathrm{TeV}
$$

We will refine this analysis by performing a full precision fit in the next section, but it is clear that the dominant driver of a light $Z^{\prime}$ is the $T$ parameter, and a $Z^{\prime}$ mass close to $3 \mathrm{TeV}$ is necessary for a good fit.

How does this result depend on the model choices made? As already remarked the dijet cross section does not make much difference, and therefore choosing non-universal couplings of the $W^{\prime}$ to the first two families (which only enter through the dijet cross section) or including a significant coupling to the third generation makes only a small difference. We might also contemplate other decay modes of the $W^{\prime}$, such as to leptons (with a light righthanded neutrino), Higgs scalars, or new fermions. Such decays would lower $B_{j j}$ which in turn requires a lighter $Z^{\prime}$. The $Z^{\prime}$ mass is bounded from below, $M_{Z^{\prime}} \geq \sqrt{2} M_{W^{\prime}} \simeq 2.69 \mathrm{TeV}$, and as $B_{j j}$ gets very small the $Z^{\prime}$ mass approaches this value.

More significantly, we might have chosen the breaking of $\mathrm{SU}(2)_{M} \times \mathrm{U}(1)_{X}$ through an $R$ dimensional representation other than a triplet. Choosing a doublet would give a lighter $Z^{\prime}$, but a somewhat worse precision fit. In addition such a light $Z^{\prime}$ comes with restrictive direct experimental bounds, predominantly from the $Z^{\prime}$ decay to leptons. Evading these bounds requires some fine tuning. For these reasons we prefer the triplet. Higher dimensional representations are also possible, and yield good precision fits with larger $Z^{\prime}$ masses, although with larger coupling $g_{M}$.

Finally we may consider including a significant vev for the alternate Higgs representation, $H_{X}$. The total vev squared of all Higgs doublets is fixed at $246 \mathrm{GeV}$, and including a larger vev for $H_{X}$ necessitates decreasing the vev for the bidoublets. Since the diboson decay of the $W^{\prime}$ comes only from the bidoublet vevs, a large diboson rate precludes a large value for the $H_{X}$ vev. Consequently the presence of this vev has only a small effect on the $Z^{\prime}$ mass. As we will see in our full model fits the trend is to push the $Z^{\prime}$ mass to the low end of the range (1.16).

\section{$2 \quad$ Symmetry breaking}

In this section we summarize the properties of the scalar fields with vacuum expectation values that result in spontaneous breaking of the gauge invariances. For the right-handed $W^{\prime}$ physics that we are considering there are two categories of scalars: those that break $\mathrm{SU}(2)_{M} \times \mathrm{U}(1)_{X} \rightarrow \mathrm{U}(1)_{Y}$ at the high scale $f$, allowing the $W^{\prime}$ and $Z^{\prime}$ to acquire large masses, and those that implement the $\mathrm{SM}$ breaking $\mathrm{SU}(2)_{\mathrm{SM}} \times \mathrm{U}(1)_{Y} \rightarrow \mathrm{U}(1)_{Q}$ at the scale $v$. 
The breaking at the scale $f$ is accomplished by a (set of) complex scalar(s) $H_{X M}$ transforming as $(1, R)_{(R-1) / 2}$ under $\left(\mathrm{SU}(2)_{L}, \mathrm{SU}(2)_{M}\right)_{\mathrm{U}(1)_{X}}$. Here $R$ is the dimension of the isospin representation of $\mathrm{SU}(2)_{M}$ and the $\mathrm{U}(1)_{X}$ charge is adjusted to preserve the conventionally chosen hypercharge generator $Y=T_{M}^{3}+X$. For any $R>1$ the unbroken gauge group is the Standard Model. A conventional normalization for the vacuum expectation values leads to masses for the $W^{\prime}$ and $Z^{\prime}$

$$
\begin{aligned}
& M_{W^{\prime}}^{2}=\frac{g_{M}^{2}}{4} \sum_{R>1} f_{R}^{2}=\frac{g^{\prime 2}}{4 \sin ^{2} \theta_{M}} \sum_{R>1} f_{R}^{2} \\
& M_{Z^{\prime}}^{2}=\frac{g_{M}^{2}+g_{X}^{2}}{4} \sum_{R>1}(R-1) f_{R}^{2}=\frac{g^{\prime 2}}{4 \sin ^{2} \theta_{M} \cos ^{2} \theta_{M}} \sum_{R>1}(R-1) f_{R}^{2} .
\end{aligned}
$$

We limit ourselves to $\mathrm{SU}(2)_{M}$ doublet and triplet representations. One of our results is that the precision fit prefers triplet breaking so that $M_{Z^{\prime}}=\sqrt{2} M_{W^{\prime}} / \cos \theta_{M}$. However a doublet is needed to adequately account for quark masses and Yukawa couplings. Consequently we will include both representations in our models, with a small doublet vev that modifies this mass relation by a few percent.

The subsequent breaking of EW symmetry at the scale $v$ must come (predominantly) from fields that transform as doublets under $\mathrm{SU}(2)_{\mathrm{SM}}$ with hypercharge $\pm 1 / 2$. As discussed earlier there are two small representations of the full gauge theory that contain Higgs doublets following the breaking at the scale $f$, and we include them both: fields $H_{X}$ transforming as $(2,1)_{\frac{1}{2}}$ and fields $H_{M}$ transforming as $(2,2)_{0}$. Both fields transform as ordinary Higgs doublets under the SM gauge group and preserve the usual leading order mass relation $M_{Z}=M_{W} / \cos \theta_{W}$. However the two types of representations have different couplings to the $W^{\prime}$ and $Z^{\prime}$. We have already discussed the consequences of this for $W W^{\prime}$ and $Z Z^{\prime}$ mixing and the associated effects on the $T$ parameter. Integrating out the heavy gauge bosons yields additional dimension six operators that are sensitive to the choice of representation and contribute to the precision electroweak fit. The relevant couplings of both types of Higgs doublets to the $W^{\prime}$ and $Z^{\prime}$ are given in (1.10).

\section{$3 \quad$ Fermion masses and mixings}

Obtaining satisfactory predictions for the SM fermion masses and CKM matrix without also generating excessive flavor changing neutral current (FCNC) couplings and meson antimeson mixings is notoriously difficult in models with right-handed $\mathrm{SU}(2)$ gauge bosons. $\mathrm{CP}$ violation in Kaon mixing leads to especially strong constraints on the couplings of the new states to first and second family quarks. Most dangerous are FCNC couplings of the $Z^{\prime}$, the Higgs, and box diagrams with exchange of one $W$ and one $W^{\prime}$ boson involving first and second family quarks.

These dangerous flavor changing effects can be avoided altogether if the right-handed SM quarks are singlets under $\mathrm{SU}(2)_{M}$, in which case fermion masses and mixings may be introduced through Yukawa couplings exactly as in the SM. The gauge couplings of the fermions preserve a full $\mathrm{U}(3)^{5}$ flavor symmetry and flavor violation enters only through 
these Yukawa couplings, also exactly as in the SM. However explaining the diboson anomaly requires a significant $W^{\prime}$ coupling to first family quarks in order to adequately produce the $W^{\prime}$. Thus the up and down quark must be at least partially contained in a doublet of $\mathrm{SU}(2)_{M}$. First family quark couplings to $W^{\prime}$ and $Z^{\prime}$ bosons are then proportional to mixing angles of quark singlets with these doublets. In order to minimize FCNCs relevant to Kaon physics we assume that these mixing angles respect (at least) SU(2)-flavor symmetries acting on the first and second family quarks.

For our precision fits the details of the fermion mass and Yukawa terms in the Lagrangian are largely irrelevant: we only need the couplings of fermions to the $W^{\prime}$ and the $Z^{\prime}$. These are determined by the fermion charges and the fraction of each SM quark that is $\mathrm{SU}(2)_{M}$ doublet. Introducing mixing angles for each SM fermion $s_{f} \equiv \sin \theta_{f}$, (where $s_{f}=0$ corresponds to pure $\mathrm{SU}(2)_{M}$ singlet fermions), these couplings take a simple generic form. The coupling of the $W^{\prime}$ to the $\mathrm{SU}(2)_{\mathrm{SM}}$ singlet up and down quarks is

$$
s_{u} s_{d} g_{M}=s_{u} s_{d} \frac{g^{\prime}}{\sin \theta_{M}},
$$

and equivalently for $(c, s)$ and $(t, b)$. We assume that $\mathrm{SU}(2)_{\mathrm{SM}}$ singlet neutrinos are heavy and there are no relevant couplings of the $W^{\prime}$ to the SM leptons. For the $Z^{\prime}$ coupling to a SM fermion field $f$ we have

$$
g_{f}=\frac{g^{\prime}}{\sin \theta_{M} \cos \theta_{M}}\left(s_{f}^{2} T_{M}^{3}-\sin ^{2} \theta_{M} Y\right)
$$

where $s_{f}$ is the fermion mixing angle, $T_{M}^{3}$ is the $\mathrm{SU}(2)_{M}$ isospin of the fermion field $f$ and $Y$ is the usual SM hypercharge.

In the following we describe two example models with different flavor symmetries. Obtaining a large top quark mass in extensions of the SM is often a challenge, and our first model will treat the third family differently from the first two. For the first two families we implement an approximate SU(2) flavor symmetry and obtain the SM quark masses through couplings to the $H_{X}$ field which has a small vev, while for the top quark we couple to the bidoublet fields with their larger vevs.

Our second model realizes the top mass through coupling only to the field $H_{X}$ and we impose an approximate SU(3) flavor symmetry on all three families of quarks. As we will see, this model also provides an excellent fit to the data, albeit at the expense of larger coupling constants and some modest tuning of parameters.

While we do not give a specific implementation for the lepton sectors, it is straightforward to extend the kind of structures we present for the quarks to leptons. In both models we will assume a separate approximate SU(3) flavor symmetry on the leptons and then, for the purposes of this paper, the only parameter that enters the lepton phenomenology is a universal lepton mixing angle.

\section{$3.1 \mathrm{SU}(2)$ flavor model}

In our first model we take both up- and down-type anti-quarks to be admixtures of $\mathrm{SU}(2)_{M}$ singlets and doublets, and assume that this mixing respects $\mathrm{SU}(2)$ flavor symmetries acting 


\begin{tabular}{|c|c|c|c|r|}
\hline & $\mathrm{SU}(3)_{c}$ & $\mathrm{SU}(2)_{L}$ & $\mathrm{SU}(2)_{M}$ & $\mathrm{U}(1)_{X}$ \\
\hline$q$ & 3 & 2 & 1 & $\frac{1}{6}$ \\
$U^{c}$ & $\overline{3}$ & 1 & 1 & $-\frac{2}{3}$ \\
$D^{c}$ & $\overline{3}$ & 1 & 1 & $\frac{1}{3}$ \\
$Q$ & 3 & 1 & 2 & $\frac{1}{6}$ \\
$Q^{c}$ & $\overline{3}$ & 1 & 2 & $-\frac{1}{6}$ \\
\hline
\end{tabular}

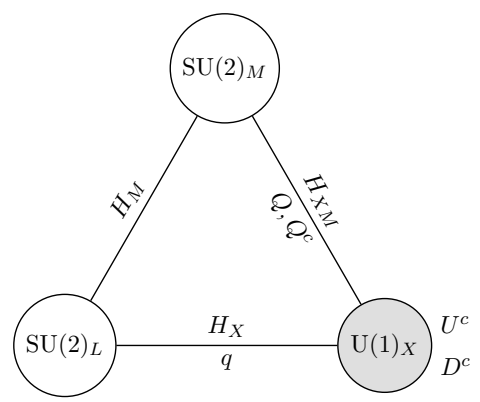

Table 1. Fields and charges for the SU(2) flavor model. All fields are left-handed.

on the first and second family anti-quark fields. We treat the third family separately, making the top quark pure $\mathrm{SU}(2)_{M}$ doublet and the bottom quark pure singlet, easily accommodating a large top quark mass.

The quarks of the first two families along with their vector-like partners are described by the fields given in table 1. Yukawa couplings and masses for the heavy fermions stem from the Lagrangian

$$
\mathcal{L} \supset y^{u} q H_{X} U^{c}+y^{d} q \tilde{H}_{X} D^{c}+Y^{u} Q H_{X M} U^{c}+Y^{d} Q \tilde{H}_{X M} D^{c}+m Q Q^{c} .
$$

Here $H_{X M}$ is an $\mathrm{SU}(2)_{M}$ doublet whose vev is at the TeV scale. The mass scale $m$ is also assumed to be at the $\mathrm{TeV}$ scale. At this scale a linear combination of the fields $\left(U^{c}, D^{c}\right)$ and $Q^{c}$ obtain a large Dirac mass with the field $Q$ from the last three terms in (3.3). Assuming that these terms respect the $\mathrm{SU}(2)$ flavor symmetry the mixing angle which parameterizes this linear combination is universal for the first two families. The orthogonal linear combinations of $\left(U^{c}, D^{c}\right)$ and $Q^{c}$ correspond to the SM anti-quarks. They obtain their Yukawa couplings to $q$ and the EW breaking Higgs doublet $H_{X}$ from the first two terms. Since the masses of the first two families are very small, the vev of $H_{X}$ can be a subdominant source of EW breaking $v_{X} \ll v$. This allows the majority of the breaking to come from the vevs of the bidoublets $H_{M}$ which determine the vector boson mixing parameters $\kappa_{W}$ and $\kappa_{Z}$. We may then use these large bidoublet vevs to obtain the top quark mass.

The third family quarks and their masses arise from the additional Lagrangian

$$
\mathcal{L} \supset y^{t} q_{3} H_{M} Q_{3}^{c}+y^{b} q_{3} \tilde{H}_{X} D_{3}^{c}+y^{Q} D_{3} \tilde{H}_{X M} Q_{3}^{c} .
$$

Here $q_{3}, Q_{3}^{c}$, and $D_{3}^{c}$ are third family copies of the fields we have included for the first two families, $D_{3}$ is a new field for the third family, and we do not include a $U_{3}^{c}$ or $Q_{3}$ field. ${ }^{4}$ The top quark acquires a mass from the first term, while the last term gives a large Dirac mass for the pair $D_{3}$ with the lower component of $Q_{3}^{c}$. Consequently the bottom anti-quark is mostly the $\mathrm{SU}(2)_{M}$ singlet field $D_{3}^{c}$, and the mixing angle for the $b$ quark is negligible. Thus we have

$$
s_{u}=s_{c}, \quad s_{d}=s_{s}, \quad s_{t}=1, \quad s_{b}=0, \text { and } s_{e}=s_{\mu}=s_{\tau} .
$$

\footnotetext{
${ }^{4}$ This third family field content is anomalous. The anomalies can be canceled with additional fields with masses at the $\mathrm{TeV}$ scale. For example, adding the set $\left\{U_{3}^{c}, D_{3}^{\prime c}, Q_{3}\right\}$ would do the trick.
} 


\section{$3.2 \mathrm{SU}(3)$ flavor model}

It would be especially attractive to accommodate the large top mass in a fully $\mathrm{SU}(3)$ flavor symmetric Lagrangian of the form of (3.3) including 3 copies of all the fields in table 1. Our previous model treated the third family differently in expectation of difficulty in obtaining a large top quark mass from the small Higgs vev $v_{X}$, but it is worth exploring if a more flavor symmetric Lagrangian is viable.

Generally speaking our precision fits prefer small values of the vev $v_{X}$, as we assumed in our introductory section. However this would necessitate a large Yukawa coupling in order to realize the large top mass. To avoid potential problems with strong coupling (and to remain within the validity of our perturbative analysis) we will limit the size of the Yukawa coupling, which in turn requires a not-so-small vev $v_{X}$. But a larger value of $v_{X}$ implies smaller bidoublet vevs, which reduces the coupling of the $W^{\prime}$ to $W Z$. To compensate for this effect we are forced to larger values of the $\mathrm{SU}(2)_{M}$ coupling, $g_{M}$. Consequently we need a compromise between large top Yukawa and large $g_{M}$. The details of this compromise will be explored in our precision fits.

There are then three relevant mixing angles for this $\mathrm{SU}(3)$ flavor symmetric model:

$$
s_{u}=s_{c}=s_{t}, \quad s_{d}=s_{s}=s_{b}, \text { and } s_{e}=s_{\mu}=s_{\tau} .
$$

\section{Fit to precision electroweak and LHC data}

Here we consider a simultaneous fit of our models to precision electroweak data and the diboson signal. We also include bounds from $W^{\prime}$ decay to dijet resonance searches and from $Z^{\prime}$ decay to dilepton resonance searches. The fit confirms and validates our simplified analysis in the Introduction.

The focus of our paper is the diboson signal and we therefore constrain the parameters of our model to produce a fixed diboson cross section at $8 \mathrm{TeV}, \sigma_{W Z}$. The remaining data is incorporated by minimizing a global $\chi^{2}$ function

$$
\chi_{\text {total }}^{2}=\chi_{l l}^{2}+\chi_{j j}^{2}+\chi_{P E W}^{2} .
$$

The values and choices we have made for each of these is detailed below.

$\sigma_{W Z}$ diboson cross section: In order to reproduce the observed diboson signal from Run 1 we fix the $W^{\prime}$ mass to $1.9 \mathrm{TeV}$ and the cross section times branching fraction to $W Z$ to $\sigma_{W Z}(8 \mathrm{TeV})=4 \mathrm{fb}$. Values of $M_{W^{\prime}}$ within the range $1.8-2.0 \mathrm{TeV}$ give similarly good fits to both the diboson data [12] and the overall $\chi_{\text {total }}^{2}$. The best fit value for $\sigma_{W Z}$ from Run 1 is in tension with the $95 \%$ confidence level upper bound obtained in Run $2 \sigma_{W Z}<25 \mathrm{fb}$ (see [12, 49] for a summary of the Run 2 searches for $W^{\prime} \rightarrow W Z$ and $\left.W^{\prime} \rightarrow W h[37-41,50]\right)$. To translate this bound into an equivalent $8 \mathrm{TeV}$ cross section bound we use a six-fold parton luminosity scaling from $8 \mathrm{TeV}$ to $13 \mathrm{TeV}$, yielding our target value for the cross section of $4 \mathrm{fb}$.

$\chi_{\text {ll }}^{2}$ dileptons from $Z^{\prime}$ decay: In Run 1 both ATLAS [51] and CMS [52] searched for the decay of a narrow resonance to dileptons. In CMS no events were seen above $1.9 \mathrm{TeV}$. 
Combining the searches for dimuons and dielectrons with assumed lepton-flavor universality, CMS obtained a $95 \%$ confidence upper limit of $0.09 \mathrm{fb}$ on the cross section times branching fraction to one species of dileptons. ATLAS saw no events above $2 \mathrm{TeV}$ and obtained a bound of $0.2 \mathrm{fb}$ for the same observable. Assuming Poisson statistics with zero observed events and combining the two bounds into a single Run 1 likelihood $\mathcal{L}=e^{-N_{C M S}} e^{-N_{A T L A S}}$ allows us to define an equivalent $\chi^{2}=-2 \log \mathcal{L}$

$$
\chi_{l l}^{2}=2\left(N_{C M S}+N_{A T L A S}\right)=6\left(\frac{\sigma_{l l}}{0.09 \mathrm{fb}}+\frac{\sigma_{l l}}{0.2 \mathrm{fb}}\right)=100 \mathrm{fb}^{-1} \sigma_{l l} .
$$

Here we used the fact that with Poisson statistics zero observed events gives a $95 \%$ confidence bound on the number of expected events $N^{95 \%}=-\log (0.05) \simeq 3$, irrespective of the number of expected background events.

$\chi_{j j}^{2}$ dijet events: Run 1 data from both CMS and ATLAS showed an intriguing $\sim 2 \sigma$ excess of dijet events with dijet invariant mass near $1.9 \mathrm{TeV}$. This data could arise from the $W^{\prime}$ decaying to dijets with a cross section of $\sigma_{j j}(8 \mathrm{TeV}) \sim 50-100 \mathrm{fb}[17,20,25,30]$. Unfortunately, neither CMS [53] nor ATLAS [42] confirmed this excess in Run 2 and instead set bounds, with the stronger bound coming from ATLAS. In our model the $W^{\prime}$ width is less than the energy resolution in ATLAS, and using an acceptance times efficiency of $\sim 50 \%$ the limit is $\sigma_{j j}(13 \mathrm{TeV}) \lesssim 150 \mathrm{fb}$. Translating this bound into an equivalent $8 \mathrm{TeV}$ bound by multiplying by first generation $\bar{q} q$ parton luminosity ratios we obtain $\sigma_{j j}(8 \mathrm{TeV}) \lesssim 24 \mathrm{fb}$ at $95 \%$ confidence. The fermion mixing angles that govern the coupling of the $W^{\prime}$ to quarks $g_{u d}=s_{u} s_{d} g_{M}$ allow accommodation of this bound. However, reducing the dijet branching fraction of the $W^{\prime}$ below that of the diboson branching fraction would require fine-tuning of parameters (see the discussion after eq. (1.15)). In order to disfavor this fine-tuned region of parameter space and motivated by the preference for dijets from Run 1 we include a non-zero central value for the dijet rate in our fit $\sigma_{j j}(8 \mathrm{TeV})=12 \pm 6 \mathrm{fb}$. The uncertainty is chosen so that the $2 \sigma$ upper bound coincides with the ATLAS $95 \%$ confidence limit. Thus we take

$$
\chi_{j j}^{2}=\left(\frac{\sigma_{j j}(8 \mathrm{TeV})-12 \mathrm{fb}}{6 \mathrm{fb}}\right)^{2}
$$

$\chi_{P E W}^{2}$ precision electroweak observables: We include all precision electroweak observables listed in the most current review of the Particle Data Group [48]. Most important in this list are the masses, widths, and line shapes of the $W$ and $Z$, precision measurements of the fermion couplings in $Z$ decay branching fractions and forward-backward asymmetries. This fit is conveniently implemented by using the work of Han and Skiba [54] who combined all constraints from precision electroweak measurements into a single $\chi_{P E W}^{2}$. We will describe this formalism and our modifications in the following.

We updated the precision electroweak function $\chi_{P E W}^{2}$ of [54] to include the best fit Higgs mass and the latest values for precision observables from the Particle Data Group [48]. The Han and Skiba $\chi_{P E W}^{2}$ function depends on the coefficients of universal dimensions 6 
operators obtained by integrating out new physics heavier than the electroweak scale. Thus to apply the formalism to our model we integrate out the $W^{\prime}$ and $Z^{\prime}$ and extract the coefficients of the dimension 6 operators so generated. Since the three families of fermions have different $W^{\prime}$ and $Z^{\prime}$ couplings in our models we generalize the operator basis in [54] to allow for non-universal operator coefficients (for similar such generalizations see $[55,56]$ ).

In the notation of [54] the operator coefficients are

$$
a_{h}=-\frac{\left(2 g_{h}\right)^{2}}{2 M_{Z^{\prime}}^{2}}+\frac{\left(g^{\prime} / \sin \theta_{M}\right)^{2}}{2 M_{W^{\prime}}^{2}} t^{4}, \quad a_{h f}=-\frac{g_{h} g_{f}}{M_{Z^{\prime}}^{2}}, \quad a_{f f^{\prime}}=-\frac{g_{f} g_{f^{\prime}}}{M_{Z^{\prime}}^{2}}
$$

where

$$
g_{h} \equiv \frac{g^{\prime}}{2 \sin \theta_{M} \cos \theta_{M}}\left(\cos ^{2} \theta_{M}-\sin ^{2} \theta_{X}\right),
$$

the fermion couplings $g_{f}$ were defined in (3.2) in terms of the charges $T_{M}^{3}$ and $Y$, and $\sin \theta_{X} \equiv v_{X} / v$ is the fraction of the EW breaking vev coming from the $H_{X}$ vev. Note that the operators in [54] are written in terms of right-handed fields for $\mathrm{SU}(2)_{\mathrm{SM}}$ singlets, $u_{R}, d_{R}, e_{R}$, and with this convention the charges for the SM fields are

\begin{tabular}{c|ccccc} 
fermion field $f$ & $q$ & $u_{R}$ & $d_{R}$ & $l$ & $e_{R}$ \\
\hline$T_{M}^{3}$ & 0 & $\frac{1}{2}$ & $-\frac{1}{2}$ & 0 & $-\frac{1}{2}$ \\
$Y$ & $\frac{1}{6}$ & $\frac{2}{3}$ & $-\frac{1}{3}$ & $-\frac{1}{2}$ & -1
\end{tabular}

No other operators are generated at tree level. We will find that the fit prefers couplings of order 1 or smaller and loop-generated operators can be neglected. The operators generated from integrating out the $Z^{\prime}$ are easily recognized as they are proportional to $1 / M_{Z^{\prime}}^{2}$. These operators all involve contractions of $\mathrm{SU}(2)_{\mathrm{SM}}$-singlet currents, and the triplet operators in the Han-Skiba basis have vanishing coefficients in our model. Integrating out the $W^{\prime}$ generates a contribution to $a_{h}$ (i.e. the $T$-operator) as already discussed in the Introduction. All other dimension six operators mediated by the $W^{\prime}$ are unimportant for several reasons: $i$. leptonic operators involve the right-handed neutrinos which we assume to be too heavy to be relevant to precision physics, ii. operators with only quarks are not sufficiently well constrained by data, and iii. operators which lead to effective couplings of righthanded fermions to the $W$ do not have an SM counterpart to interfere with. Therefore their contributions to observables are as small as contributions from dimension 8 operators which we have consistently ignored.

In addition to the usual SM couplings and the $W^{\prime}$ mass (fixed to $M_{W^{\prime}}=1.9 \mathrm{TeV}$ ) both our models have the following continuous free parameters

$$
\cos \theta_{M}, t^{2}, s_{u}, s_{d}, s_{e}, \sin \theta_{X}
$$

Fits for the $\mathrm{SU}(2)$ flavor model will prefer very small values of $\sin \theta_{X}$ and consequently this angle plays little role in our analysis of this model. In the SU(3) model the fits also prefer small values of $\sin \theta_{X}$. However, in this case the top quark mass is given by $m_{t}=y_{t} c_{u} v \sin \theta_{X} / \sqrt{2}$ so that small $\sin \theta_{X}$ requires large $y_{t}$ to compensate. In order to remain safely in the perturbative part of parameter space we impose the constraint $y_{t} \leq 2$. This limits the size of $\sin \theta_{X} \geq m_{t} /\left(c_{u} v \sqrt{2}\right)$. Since the fit prefers small values of $v_{X}$, the best 


\begin{tabular}{|c|c|c|c|c|}
\hline & $\begin{array}{c}\text { SU(2)Model } \\
k=\sqrt{2}\end{array}$ & $\begin{array}{c}\text { SU(3)Model } \\
k=\sqrt{2}\end{array}$ & $\begin{array}{c}\text { SU(2)Model } \\
k=1\end{array}$ & $\begin{array}{c}\text { SU(3)Model } \\
k=1\end{array}$ \\
\hline $\cos \theta_{M}$ & 0.92 & 0.97 & 0.87 & 0.94 \\
$t^{2}$ & 0.50 & 0.40 & 0.62 & 0.55 \\
$\left\langle H_{X}\right\rangle[\mathrm{GeV}]$ & 0 & 137 & 0 & 127 \\
$s_{d}$ & 0.32 & 0.20 & 0.37 & 0.34 \\
$s_{e}$ & 0.30 & 0.29 & 0.56 & 0.39 \\
$s_{u}$ & 0.54 & 0.54 & 0.57 & 0.42 \\
\hline$M_{Z^{\prime}}[\mathrm{TeV}]$ & 2.92 & 2.78 & 2.18 & 2.02 \\
$\sigma_{j j}(13 \mathrm{TeV})[\mathrm{fb}]$ & 74 & 66 & 71 & 62 \\
$\sigma_{l l}(13 \mathrm{TeV})[\mathrm{fb}]$ & 0.03 & 0.08 & 0.17 & 0.11 \\
\hline$\triangle \chi_{P E W}^{2}$ & -1.1 & -1.7 & 1.1 & 0.5 \\
$\triangle \chi_{\text {total }}^{2}$ & -4.9 & -5.1 & -0.8 & -2.0 \\
\hline
\end{tabular}

Table 2. Best fit points and predictions in the 4 Models for fixed $\sigma_{W Z}(8 \mathrm{TeV})=4 \mathrm{fb}$ corresponding to $\sigma_{W Z}(13 \mathrm{TeV}) \simeq 24 \mathrm{fb}$. Note that $\sin \theta_{X}=\left\langle H_{X}\right\rangle / v$.

fit point is always near the smallest possible value for $\sin \theta_{X}$. We can therefore simplify our analysis by fixing $\sin \theta_{X}$ in our fits for the $\mathrm{SU}(3)$ model to saturate this inequality: $\sin \theta_{X}=m_{t} /\left(c_{u} v \sqrt{2}\right)$.

The choice of $\mathrm{SU}(2)_{M}$ representation for the scalar field $H_{M}$ introduces an additional discrete parameter $k=1, \sqrt{2}, \sqrt{3}, \ldots$ that enters the relationship between the $Z^{\prime}$ and $W^{\prime}$ masses $M_{Z^{\prime}}=k M_{W^{\prime}} / \cos \theta_{M}$. We focus on the two simplest cases: doublet breaking with $k=1$ and triplet breaking with $k=\sqrt{2}$. Larger representations for $H_{X M}$ would lead to heavier $Z^{\prime}$ masses for which good fits to the precision electroweak data can also be obtained.

Table 2 shows the best fit parameters for the two Models and the two choices $k=1$ and $k=\sqrt{2}$. For each case we show the mass of the $Z^{\prime}$, the expected $W^{\prime}$ to dijet and $Z^{\prime}$ to dilepton rates at $13 \mathrm{TeV}$, and two different measures of the goodness of fit. The first measure is the difference between $\chi_{P E W}^{2}$ of the best fit point relative to the SM, $\left.\triangle \chi_{P E W}^{2} \equiv \chi_{P E W}^{2}\right|_{\text {best fit }}-\left.\chi_{P E W}^{2}\right|_{\mathrm{SM}}$, while the second is the difference of the overall $\chi_{\text {total }}^{2}$ relative to the SM, $\left.\triangle \chi_{\text {total }}^{2} \equiv \chi_{\text {total }}^{2}\right|_{\text {best fit }}-\left.\chi_{\text {total }}^{2}\right|_{\mathrm{SM}}$. Note that differences in $\chi_{\text {total }}^{2}$ on the order of a few should be taken with a grain of salt because of the somewhat arbitrary choice of central value for the dijet cross section in $\chi_{j j}^{2}$.

As expected the best fit points for models with $k=\sqrt{2}$ have $Z^{\prime}$ masses larger than those for models with $k=1$, and therefore more easily avoid constraints from both direct searches for $Z^{\prime} \rightarrow l \bar{l}$ in Run 1 and Run 2 and precision electroweak measurements. We further explore models with $k=\sqrt{2}$ in the next subsection. While the models with $k=1$ have a significantly worse $\triangle \chi^{2}$ compared to the $k=\sqrt{2}$ models, a small region of parameter space which satisfies all constraints exists. We discuss this case following the $k=\sqrt{2}$ analysis. 

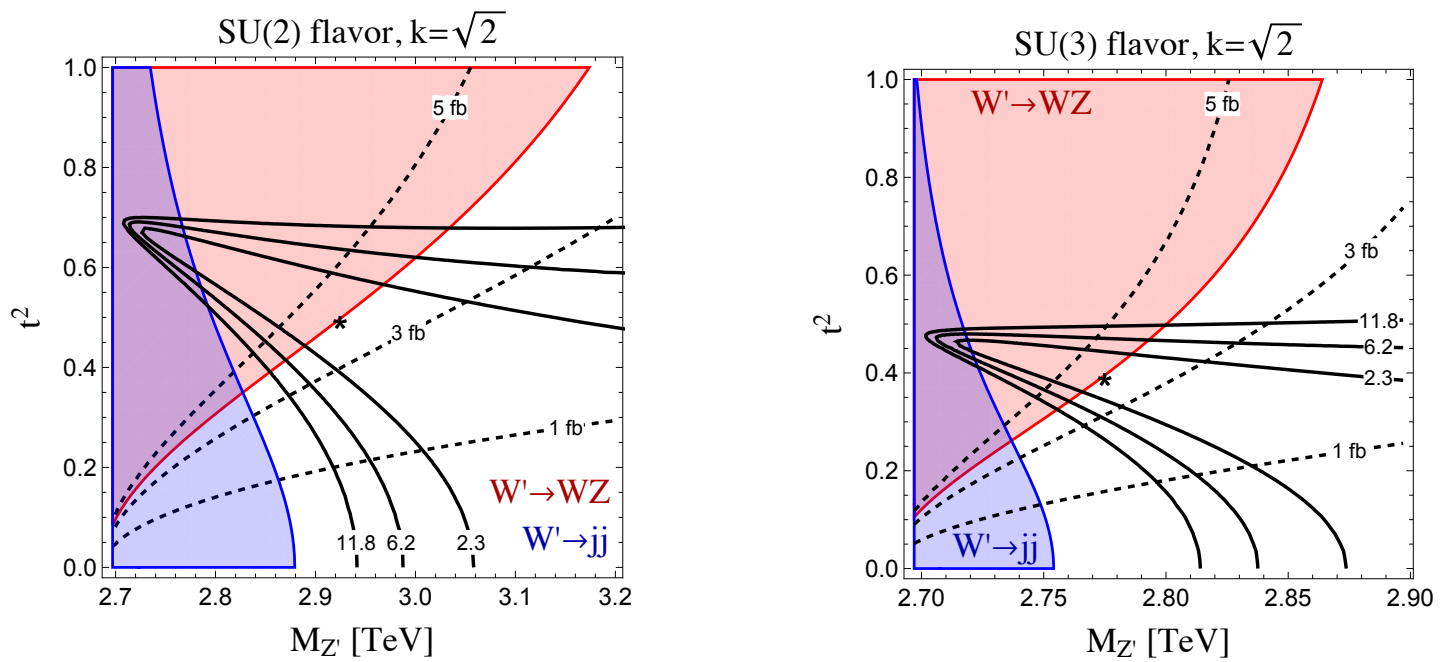

Figure 2. Preferred and excluded regions in the $M_{Z^{\prime}}$ versus $t^{2}$ parameter space in SU(2) and SU(3) flavor models with $k=\sqrt{2}$. The fermion mixing parameters $s_{e}, s_{d}, s_{u}$ are fixed at the best fit values in table 2. Solid lines are contours of constant $\triangle \chi_{P E W}^{2}$, enclosing $68 \%, 95 \%$, and $99.7 \%$ confidence regions in the Gaussian approximation. Dashed lines are contours of constant $8 \mathrm{TeV} \mathrm{WZ}$ diboson cross section. Colored regions are excluded by diboson searches from ATLAS and CMS at $13 \mathrm{TeV}$ and dijet resonance searches from ATLAS and CMS at $13 \mathrm{TeV}$. The best fit points of table 2 are indicated by asterisks. A satisfactory PEW fit with a sizable diboson cross section (for example $\sigma_{W Z} \gtrsim 3 \mathrm{fb}$ ) fixes the $Z^{\prime}$ mass to lie near $3.0 \mathrm{TeV}$ in the $\mathrm{SU}(2)$ model and near $2.8 \mathrm{TeV}$ in the $\mathrm{SU}(3)$ model.

\subsection{Models with triplet breaking $k=\sqrt{2}$}

Both models with $k=\sqrt{2}$ allow excellent fits, obtaining the diboson and dijet signals while avoiding constraints from $Z^{\prime} \rightarrow l \bar{l}$ searches. Both also have precision electroweak fits that improve upon the SM. In all cases the $Z^{\prime}$ mass is predicted to be near the range 2.8-3.0 TeV and out of reach of the Run 1 dilepton search for generic values of the fermion mixing angles.

To understand the robustness of the fits and explore the parameter spaces of the two $k=\sqrt{2}$ models we plot the main LHC observables and $\chi_{P E W}^{2}$ as a function of the model parameters $M_{Z^{\prime}}=\sqrt{2} / \cos \theta_{M} \times 1.9 \mathrm{TeV}$ and $t^{2}$ in figure 2 . In these plots we hold the remaining parameters fixed to their best fit values shown in table 2 . The colored regions in the plots correspond to the direct $95 \%$ confidence search limits from Run 1 and Run 2 at the LHC. The $Z^{\prime} \rightarrow l \bar{l}$ searches place no restrictions on the parameter space shown. However, both dijet and diboson searches exclude significant portions of this parameter space. We also plot contours of constant $\triangle \chi_{P E W}^{2}$ relative to the point which minimizes $\chi_{P E W}^{2}$. In the Gaussian approximation the contours labeled 2.3,6.2, 11.8 then correspond to $68 \%, 95 \%, 99.7 \%$ confidence regions in this two-dimensional parameter space.

For each model a large region of parameter space satisfies both precision electroweak constraints and direct searches. Requiring a sizeable $8 \mathrm{TeV}$ diboson signal narrows the allowed region to a small domain near the best fit point. Within this domain the $Z^{\prime}$ mass is predicted to lie near $2.8-3.0 \mathrm{TeV}$. 
Not visible in these plots is a somewhat flat direction for $\chi_{\text {total }}^{2}$ along the axis of the wedge-shaped region bounded by the precision electroweak contours. In moving along this trough in parameter space the hidden parameters $s_{d}, s_{u}$ can be adjusted to avoid the constraints from diboson and dijet searches. For smaller values of $M_{Z^{\prime}}$ the trough gets increasingly narrow. This is a sign that $t^{2}$ must be finely tuned to maintain a good PEW fit. For larger values of $M_{Z^{\prime}} \gtrsim 3.1 \mathrm{TeV}$ the combined requirement of a sizeable diboson signal with a good PEW fit can no longer be satisfied. Thus the $Z^{\prime}$ mass prediction is quite robust, with both models requiring a $Z^{\prime}$ mass between 2.7 and $3.1 \mathrm{TeV}$.

This prediction is intriguing in light of a di-electron event with invariant mass of $\simeq 2.9 \mathrm{TeV}$ observed by CMS in Run 2 [57]. The likelihood that this event is due to SM backgrounds is quite small: these backgrounds contribute only $0.036 \pm 0.009$ events integrated over all invariant mass greater than $2.8 \mathrm{TeV}$ [57]. It is therefore worthwhile asking whether our predicted $13 \mathrm{TeV}$ cross section for $Z^{\prime}$ production with subsequent $Z^{\prime} \rightarrow$ ee decay makes this process a likely explanation of the CMS event.

The predicted number of dilepton events in our model is most sensitive to the parameters $s_{u}$ and $\cos \theta_{M}$ which determine the $Z^{\prime}$ coupling to up-quarks. We therefore show the $Z^{\prime}$ relative width $\Gamma / M$ (dashed) and predicted dilepton event rates (red, solid) from $Z^{\prime} \rightarrow l \bar{l}$ decay at Run 2 of ATLAS and CMS combined as a function of these parameters in the right panels of figures 3 and 4 . One sees that as $M_{Z^{\prime}}$ decreases the gauge coupling $g_{M}$ grows and the $Z^{\prime}$ width increases. Similarly, large $s_{u}$ implies a larger decay rate to up quarks and an increased width. The solid red lines in the panel on the right indicate contours of constant total number of $Z^{\prime} \rightarrow l \bar{l}$ events predicted for CMS and ATLAS and muons and electrons combined. For the SU(2) model in the preferred region of parameter space near $M_{Z^{\prime}} \simeq 2.9 \mathrm{TeV}$, the $Z^{\prime}$ has a width of about $1 \%$ and the number of events expected at $13 \mathrm{TeV}$ varies between 0.05 and about 5 . For the $\mathrm{SU}(3)$ model, the preferred $Z^{\prime}$ mass is $M_{Z^{\prime}} \sim 2.8 \mathrm{TeV}$, and the width is also about $1 \%$ with between 0.1 and 2 events expected. Both models are perfectly consistent with the 1 observed $Z^{\prime} \rightarrow e^{+} e^{-}$event observed by CMS in Run 2 and promise many more events in the upcoming $13 \mathrm{TeV}$ runs.

\subsection{Models with doublet breaking $k=1$}

The $\mathrm{SU}(2)$ and $\mathrm{SU}(3)$ models with $k=1$ predict a relatively light $Z^{\prime}$. In both models it is possible to tune $s_{u}$ and $s_{d}$ such that the $Z^{\prime}$ coupling to quarks is very small (see eq. (3.2)). In this somewhat tuned region of parameter space the $Z^{\prime}$ production cross section sufficiently small to evade any $Z^{\prime}$ search bounds. In the $\mathrm{SU}(2)$ model the viable region corresponds to a $Z^{\prime}$ mass near $2.2 \mathrm{TeV}$ with a width well below $1 \%$. In the $\mathrm{SU}(3)$ model there is a slightly larger allowed region with $Z^{\prime}$ mass near $2.0 \mathrm{TeV}$ and also a very narrow width.

Since neither Run 1 nor Run 2 have observed dilepton events at 2.0 or $2.2 \mathrm{TeV}$ we can combine the dilepton bounds from ATLAS and CMS at 8 and $13 \mathrm{TeV}$ for both muons and electrons. ${ }^{5}$

\footnotetext{
${ }^{5}$ While there are some events — consistent with the tail of the Drell-Yan distribution — at 1.8 and $1.9 \mathrm{TeV}$, our $Z^{\prime}$ is always heavier than $M_{W^{\prime}}=1.9 \mathrm{TeV}$ and very narrow in the allowed parameter space. Therefore we discount the possibility that these events arise from $Z^{\prime}$ production.
} 

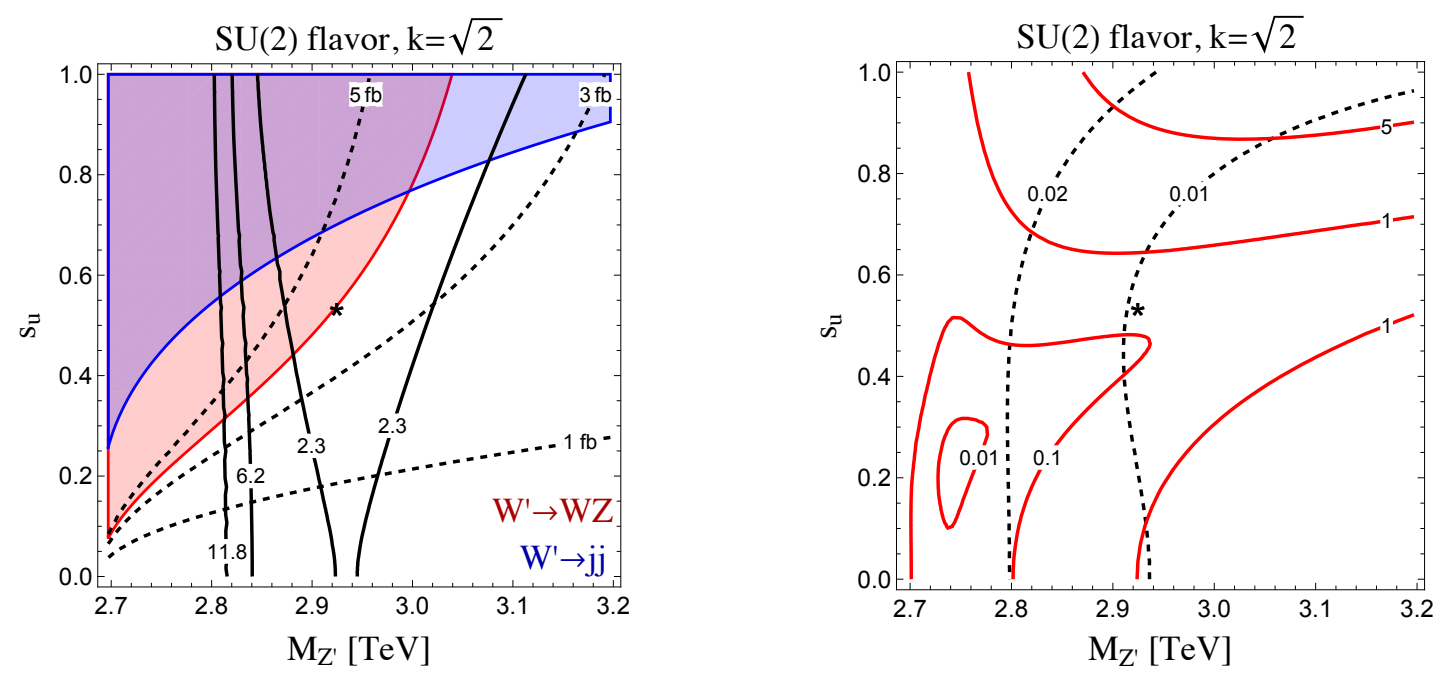

Figure 3. Preferred and excluded regions in the $M_{Z^{\prime}}$ versus $s_{u}$ plane in the SU(2) model with $k=\sqrt{2}$. The parameters $s_{e}, s_{d}, t^{2}$ are held fixed at the best fit values given in table 2 . The contours in the left plot are are as in figure 2. The plot on the right shows contours of the predicted number of dilepton $Z^{\prime} \rightarrow l \bar{l}$ events in Run 2 at CMS and ATLAS and electron plus muon final states combined (red, solid). Also shown are contours of constant $Z^{\prime}$ width over mass (dashed). Note that in the parameter region with a satisfactory PEW fit and sizeable $W^{\prime} \rightarrow W Z$ cross section the $Z^{\prime}$ width is about $1 \%$ and the predicted Run 2 dilepton event rate ranges from less than 0.1 events to 5 events.
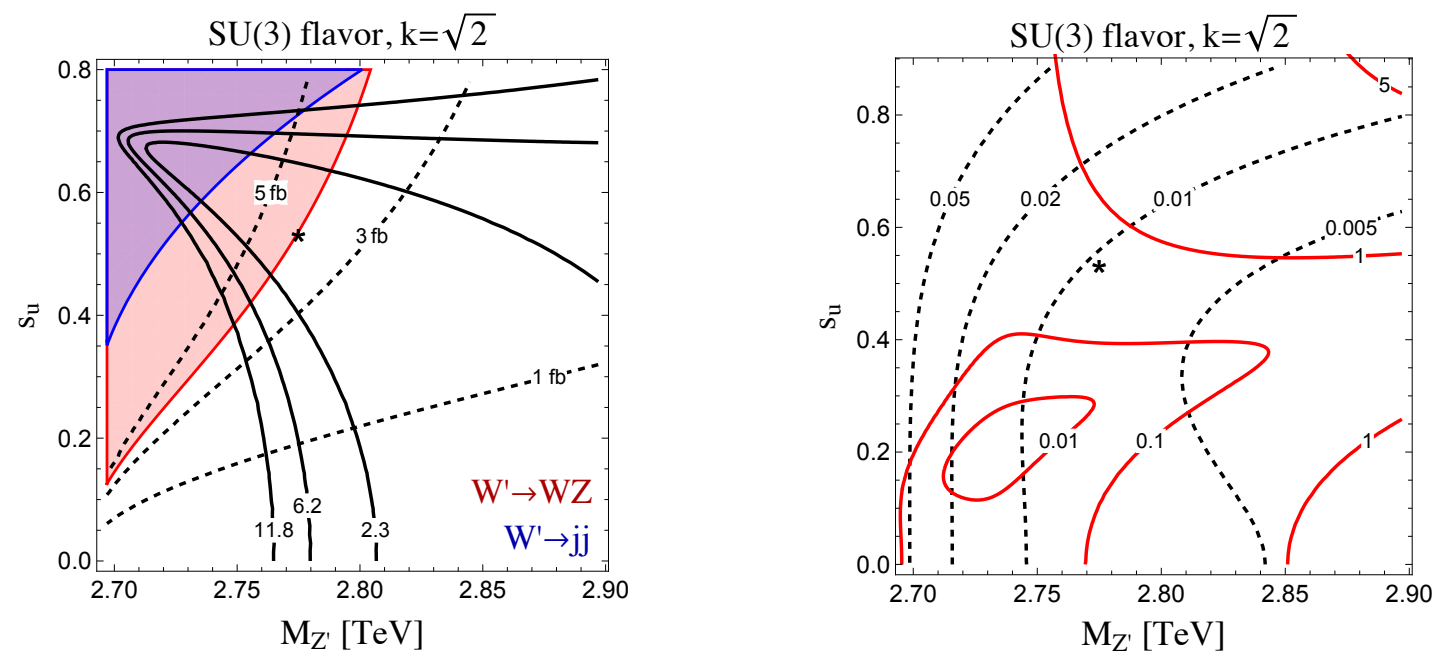

Figure 4. Preferred regions of $M_{Z^{\prime}}$ versus $s_{u}$ parameter space in the SU(3) model with predictions for the width of the $Z^{\prime}$ and dilepton event rate at Run 2. See caption for figure 3 for details.

In figure 5 we show two slices of parameter space. The plot in the right panel shows that the viable region requires significant fine tuning of both $s_{u}$ and $s_{d}$ to simultaneously avoid the dilepton bounds and obtain an interesting $W Z$ diboson signal. Figure 6 shows the $Z^{\prime}$ width and the expected Run 2 dilepton event rate along side the allowed parameter 

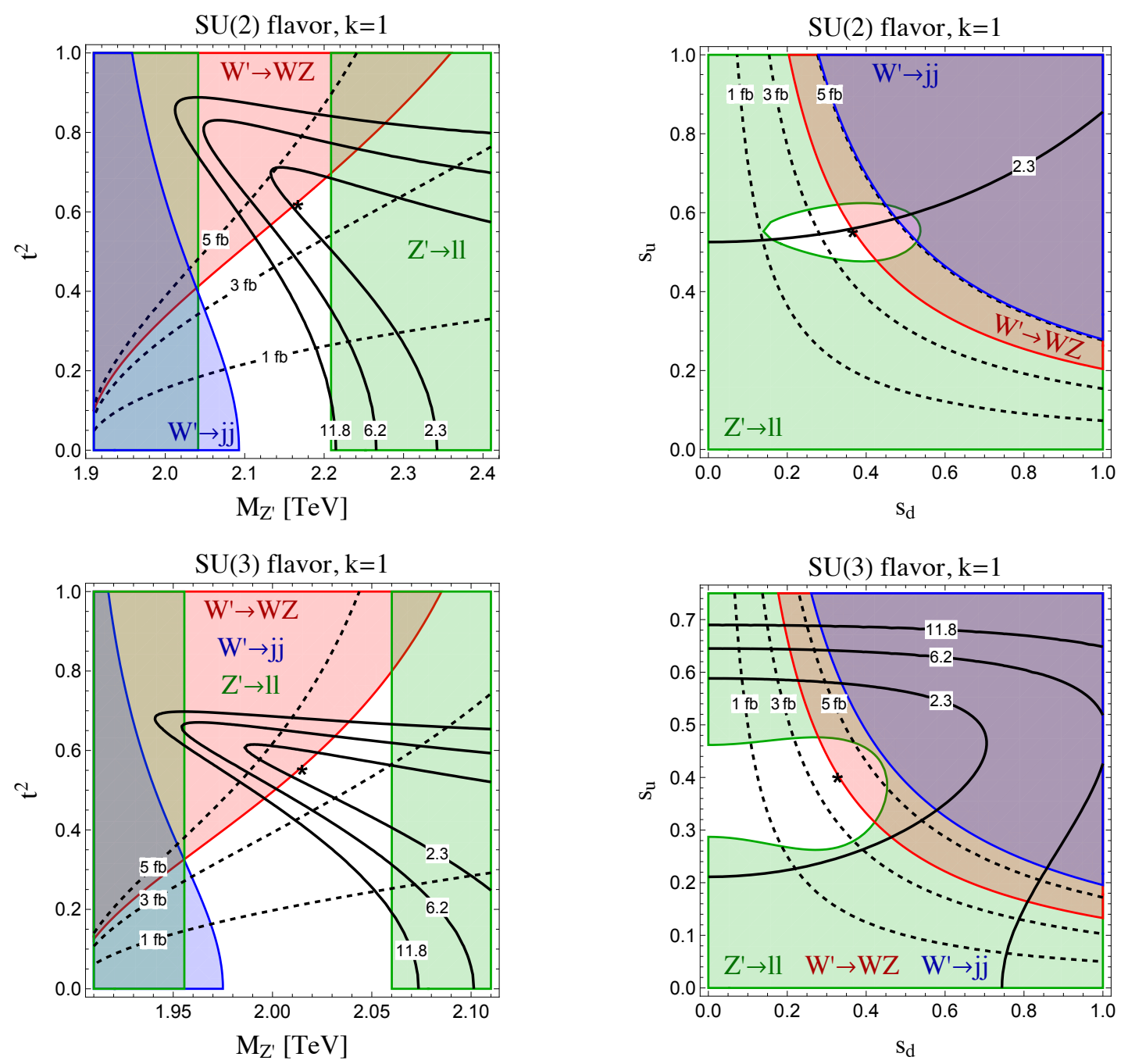

Figure 5. Preferred and excluded regions in the $M_{Z^{\prime}}$ versus $t^{2}$ and $s_{d}$ versus $s_{u}$ parameter space in the $\mathrm{SU}(2)$ and $\mathrm{SU}(3)$ models with $k=1$. Note that most of parameter space is ruled out by the dilepton searches at ATLAS and CMS at $8 \mathrm{TeV}$ and $13 \mathrm{TeV}$ combined (green). The remaining allowed region has finely tuned values for $s_{u}$ and $s_{d}$ which minimize the $Z^{\prime}$ production cross section.

spaces in the $M_{Z^{\prime}}-s_{u}$ plane for the two models. Since the $Z^{\prime}$ is very narrow in both cases and the dilepton event rate is already very close to the $95 \%$ confidence bound, both $k=1$ models will be discovered or ruled out with only a little additional $13 \mathrm{TeV}$ running.

\section{Acknowledgments}

We thank David E. Kaplan for useful discussions. This work was supported by the U.S. Department of Energy's Office of Science. 

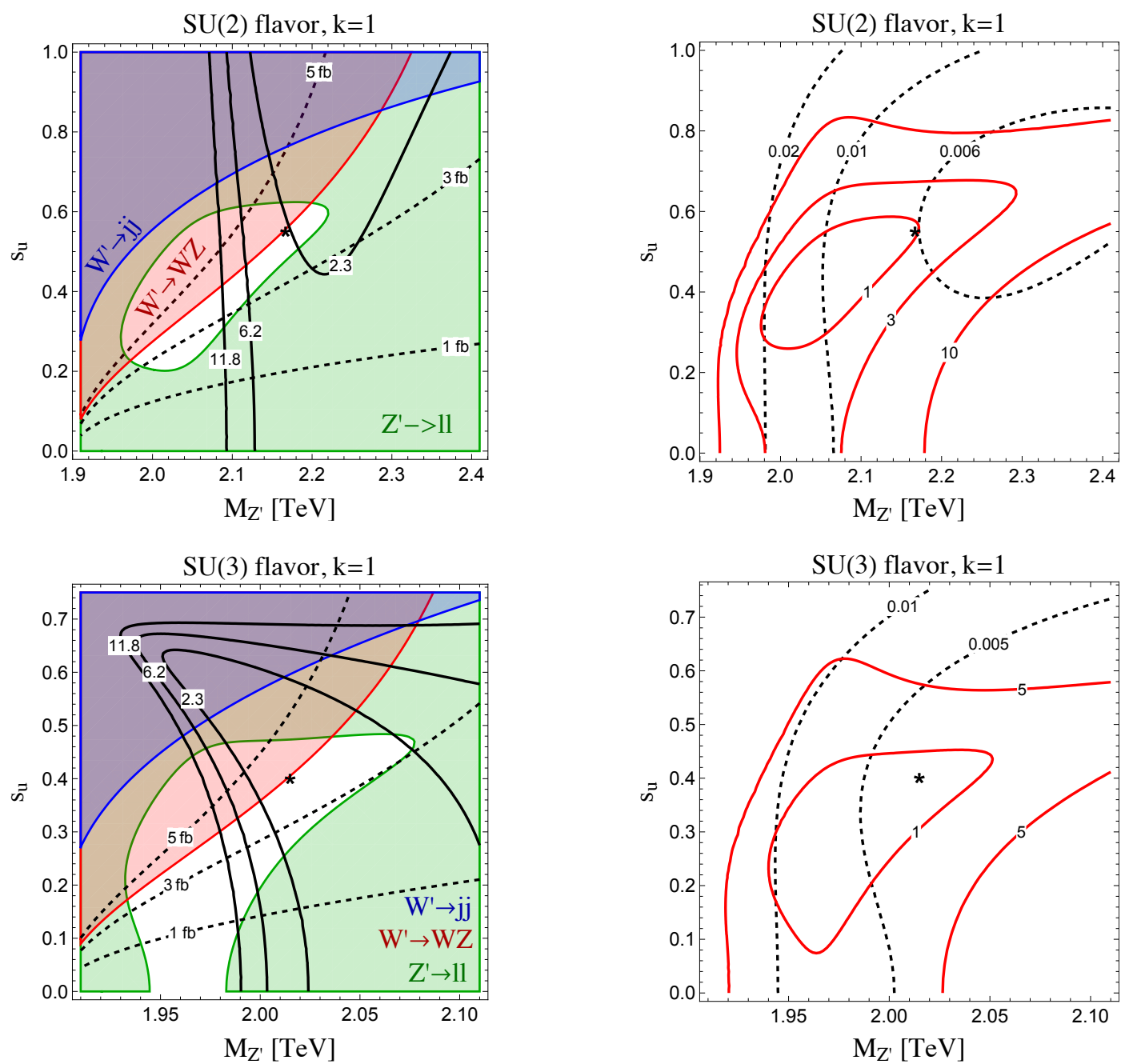

Figure 6. The plots on the left show PEW and direct search constraints in the $M_{Z^{\prime}} \quad v s . s_{u}$ parameter spaces of the $\mathrm{SU}(2)$ and $\mathrm{SU}(3)$ models for $\mathrm{k}=1$. The contour plots on the right show the predicted number of dilepton events at ATLAS and CMS combined for Run 2 (red, solid) and the predicted Z' width (black, dashed) in the same parameter space.

Open Access. This article is distributed under the terms of the Creative Commons Attribution License (CC-BY 4.0), which permits any use, distribution and reproduction in any medium, provided the original author(s) and source are credited.

\section{References}

[1] ATLAS collaboration, Search for high-mass diboson resonances with boson-tagged jets in proton-proton collisions at $\sqrt{s}=8 \mathrm{TeV}$ with the ATLAS detector, JHEP 12 (2015) 055 [arXiv: 1506.00962] [INSPIRE].

[2] ATLAS collaboration, Search for resonant diboson production in the $\ell \ell q \bar{q}$ final state in $p p$ collisions at $\sqrt{s}=8 \mathrm{TeV}$ with the ATLAS detector, Eur. Phys. J. C 75 (2015) 69 [arXiv: 1409.6190] [INSPIRE]. 
[3] ATLAS collaboration, Search for production of $W W / W Z$ resonances decaying to a lepton, neutrino and jets in pp collisions at $\sqrt{s}=8 \mathrm{TeV}$ with the ATLAS detector, Eur. Phys. J. C 75 (2015) 209 [Erratum ibid. C 75 (2015) 370] [arXiv:1503.04677] [INSPIRE].

[4] ATLAS collaboration, Search for a new resonance decaying to a $W$ or $Z$ boson and a Higgs boson in the $\ell \ell / \ell \nu / \nu \nu+b \bar{b}$ final states with the ATLAS detector, Eur. Phys. J. C 75 (2015) 263 [arXiv: 1503.08089] [INSPIRE].

[5] ATLAS collaboration, Search for new phenomena in the dijet mass distribution using $p-p$ collision data at $\sqrt{s}=8 \mathrm{TeV}$ with the ATLAS detector, Phys. Rev. D 91 (2015) 052007 [arXiv: 1407.1376] [INSPIRE].

[6] CMS collaboration, Search for massive resonances in dijet systems containing jets tagged as $W$ or $Z$ boson decays in pp collisions at $\sqrt{s}=8 \mathrm{TeV}$, JHEP 08 (2014) 173 [arXiv: 1405.1994] [INSPIRE].

[7] CMS collaboration, Search for massive resonances decaying into pairs of boosted bosons in semi-leptonic final states at $\sqrt{s}=8 \mathrm{TeV}$, JHEP 08 (2014) 174 [arXiv:1405.3447] [INSPIRE].

[8] CMS collaboration, Search for massive WH resonances decaying into the $\ell \nu \mathrm{b} \overline{\mathrm{b}}$ final state at $\sqrt{s}=8 \mathrm{TeV}$, Eur. Phys. J. C 76 (2016) 237 [arXiv:1601.06431] [INSPIRE].

[9] CMS collaboration, Search for a massive resonance decaying into a Higgs boson and a $W$ or $Z$ boson in hadronic final states in proton-proton collisions at $\sqrt{s}=8 \mathrm{Te}$, JHEP 02 (2016) 145 [arXiv: 1506.01443] [INSPIRE].

[10] CMS collaboration, Search for Narrow High-Mass Resonances in Proton-Proton Collisions at $\sqrt{s}=8$ TeV Decaying to a $Z$ and a Higgs Boson, Phys. Lett. B 748 (2015) 255 [arXiv: 1502.04994] [INSPIRE].

[11] CMS collaboration, Search for resonances and quantum black holes using dijet mass spectra in proton-proton collisions at $\sqrt{s}=8$ TeV, Phys. Rev. D 91 (2015) 052009 [arXiv: 1501.04198] [INSPIRE].

[12] F. Dias et al., Combination of Run-1 Exotic Searches in Diboson Final States at the LHC, JHEP 04 (2016) 155 [arXiv: 1512.03371] [INSPIRE].

[13] J. Brehmer et al., The Diboson Excess: Experimental Situation and Classification of Explanations; A Les Houches Pre-Proceeding, arXiv:1512.04357 [INSPIRE].

[14] J. Hisano, N. Nagata and Y. Omura, Interpretations of the ATLAS Diboson Resonances, Phys. Rev. D 92 (2015) 055001 [arXiv: 1506.03931] [INSPIRE].

[15] D.B. Franzosi, M.T. Frandsen and F. Sannino, Diboson Signals via Fermi Scale Spin-One States, Phys. Rev. D 92 (2015) 115005 [arXiv:1506.04392] [InSPIRE].

[16] K. Cheung, W.-Y. Keung, P.-Y. Tseng and T.-C. Yuan, Interpretations of the ATLAS Diboson Anomaly, Phys. Lett. B 751 (2015) 188 [arXiv:1506.06064] [InSPIRE].

[17] B.A. Dobrescu and Z. Liu, W? Boson near 2 TeV: Predictions for Run 2 of the LHC, Phys. Rev. Lett. 115 (2015) 211802 [arXiv:1506.06736] [INSPIRE].

[18] Y. Gao, T. Ghosh, K. Sinha and J.-H. Yu, SU(2) $\times \mathrm{SU}(2) \times \mathrm{U}(1)$ interpretations of the diboson and Wh excesses, Phys. Rev. D 92 (2015) 055030 [arXiv:1506.07511] [INSPIRE].

[19] A. Thamm, R. Torre and A. Wulzer, Composite Heavy Vector Triplet in the ATLAS Diboson Excess, Phys. Rev. Lett. 115 (2015) 221802 [arXiv:1506.08688] [InSPIRE].

[20] J. Brehmer, J. Hewett, J. Kopp, T. Rizzo and J. Tattersall, Symmetry Restored in Dibosons at the LHC?, JHEP 10 (2015) 182 [arXiv: 1507.00013] [INSPIRE]. 
[21] Q.-H. Cao, B. Yan and D.-M. Zhang, Simple non-Abelian extensions of the standard model gauge group and the diboson excesses at the LHC, Phys. Rev. D 92 (2015) 095025 [arXiv: 1507.00268] [INSPIRE].

[22] T. Abe, T. Kitahara and M.M. Nojiri, Prospects for Spin-1 Resonance Search at $13 \mathrm{TeV}$ LHC and the ATLAS Diboson Excess, JHEP 02 (2016) 084 [arXiv: 1507.01681] [INSPIRE].

[23] A. Carmona, A. Delgado, M. Quirós and J. Santiago, Diboson resonant production in non-custodial composite Higgs models, JHEP 09 (2015) 186 [arXiv: 1507.01914] [INSPIRE].

[24] B.C. Allanach, B. Gripaios and D. Sutherland, Anatomy of the ATLAS diboson anomaly, Phys. Rev. D 92 (2015) 055003 [arXiv: 1507.01638] [INSPIRE].

[25] B.A. Dobrescu and Z. Liu, Heavy Higgs bosons and the $2 \mathrm{TeV} W^{\prime}$ boson, JHEP 10 (2015) 118 [arXiv: 1507.01923] [INSPIRE].

[26] G.M. Pelaggi, A. Strumia and S. Vignali, Totally asymptotically free trinification, JHEP 08 (2015) 130 [arXiv: 1507.06848] [InSPIRE].

[27] K. Lane and L. Pritchett, Heavy Vector Partners of the Light Composite Higgs, Phys. Lett. B 753 (2016) 211 [arXiv: 1507.07102] [inSPIRE].

[28] A.E. Faraggi and M. Guzzi, Extra $Z^{\prime} s$ and $W^{\prime} s$ in heterotic-string derived models, Eur. Phys. J. C 75 (2015) 537 [arXiv: 1507.07406] [inSPIRE].

[29] M. Low, A. Tesi and L.-T. Wang, Composite spin-1 resonances at the LHC, Phys. Rev. D 92 (2015) 085019 [arXiv: 1507.07557] [INSPIRE].

[30] B.A. Dobrescu and P.J. Fox, Signals of a $2 \mathrm{TeV} W^{\prime}$ boson and a heavier $Z^{\prime}$ boson, arXiv: 1511.02148 [INSPIRE].

[31] A. Sajjad, Understanding diboson anomalies, Phys. Rev. D 93 (2016) 055028 [arXiv: 1511.02244] [INSPIRE].

[32] K. Das, T. Li, S. Nandi and S.K. Rai, Diboson excesses in an anomaly free leptophobic left-right model, Phys. Rev. D 93 (2016) 016006 [arXiv:1512.00190] [INSPIRE].

[33] F.F. Deppisch et al., Reconciling the $2 \mathrm{TeV}$ excesses at the LHC in a linear seesaw left-right model, Phys. Rev. D 93 (2016) 013011 [arXiv: 1508.05940] [INSPIRE].

[34] P.S. Bhupal Dev and R.N. Mohapatra, Unified explanation of the eejj, diboson and dijet resonances at the LHC, Phys. Rev. Lett. 115 (2015) 181803 [arXiv:1508.02277] [INSPIRE].

[35] A. Das, N. Nagata and N. Okada, Testing the 2-TeV Resonance with Trileptons, JHEP 03 (2016) 049 [arXiv: 1601.05079] [INSPIRE].

[36] J.H. Collins and W.H. Ng, $A 2 \mathrm{TeV} W_{R}$, supersymmetry and the Higgs mass, JHEP 01 (2016) 159 [arXiv:1510.08083] [inSPIRE].

[37] ATLAS collaboration, Search for $W W / W Z$ resonance production in the $\ell \nu q q$ final state at $\sqrt{s}=13 \mathrm{TeV}$ with the ATLAS detector at the LHC, ATLAS-CONF-2015-075 (2015).

[38] ATLAS collaboration, Search for diboson resonances in the llqq final state in pp collisions at $\sqrt{s}=13 \mathrm{TeV}$ with the ATLAS detector, ATLAS-CONF-2015-071 (2015).

[39] ATLAS collaboration, Search for resonances with boson-tagged jets in 3.2 $\mathrm{fb}^{-1}$ of $\mathrm{p} \mathrm{p}$ collisions at $\sqrt{s}=13$ TeV collected with the ATLAS detector, ATLAS-CONF-2015-073 (2015).

[40] ATLAS collaboration, Search for diboson resonances in the $\nu \nu q q$ final state in pp collisions at $\sqrt{s}=13 \mathrm{TeV}$ with the ATLAS detector, ATLAS-CONF-2015-068 (2015). 
[41] CMS collaboration, Search for massive resonances decaying into pairs of boosted $W$ and $Z$ bosons at $\sqrt{s}=13$ TeV, CMS-PAS-EXO-15-002 (2015).

[42] ATLAS collaboration, Search for new phenomena in dijet mass and angular distributions from pp collisions at $\sqrt{s}=13$ TeV with the ATLAS detector, Phys. Lett. B 754 (2016) 302 [arXiv: 1512.01530] [INSPIRE].

[43] P. Coloma, B.A. Dobrescu and J. Lopez-Pavon, Right-handed neutrinos and the 2 TeV $W^{\prime}$ boson, Phys. Rev. D 92 (2015) 115023 [arXiv:1508.04129] [INSPIRE].

[44] A.D. Martin, W.J. Stirling, R.S. Thorne and G. Watt, Parton distributions for the LHC, Eur. Phys. J. C 63 (2009) 189 [arXiv:0901.0002] [InSPIRE].

[45] Q.-H. Cao, Z. Li, J.-H. Yu and C.P. Yuan, Discovery and Identification of $W$ ' and Z' in $\mathrm{SU}(2) \times \mathrm{SU}(2) \times \mathrm{U}(1)$ Models at the LHC, Phys. Rev. D 86 (2012) 095010 [arXiv: 1205.3769] [INSPIRE].

[46] M. Carena, A. Daleo, B.A. Dobrescu and T.M.P. Tait, $Z^{\prime}$ gauge bosons at the Tevatron, Phys. Rev. D 70 (2004) 093009 [hep-ph/0408098] [INSPIRE].

[47] R. Hamberg, W.L. van Neerven and T. Matsuura, A Complete calculation of the order $\alpha_{s}^{2}$ correction to the Drell-Yan K factor, Nucl. Phys. B 359 (1991) 343 [Erratum ibid. B 644 (2002) 403] [INSPIRE].

[48] Particle Data Group collaboration, K.A. Olive et al., Review of Particle Physics, Chin. Phys. C 38 (2014) 090001 [inSPIRE].

[49] M. Bellomo, Searches for Boosted Di-Boson Resonances with the ATLAS and CMS detectors, at 51st Rencontres de Moriond on Electroweak Interactions and Unified Theories, La Thuile Italy (2016).

[50] ATLAS collaboration, Search for new resonances decaying to a $W$ or $Z$ boson and a Higgs boson in the $\ell \ell b \bar{b}, \ell \nu b \bar{b}$ and $\nu \nu b \bar{b}$ channels in pp collisions at $\sqrt{s}=13$ TeV with the ATLAS detector, ATLAS-CONF-2015-074 (2015).

[51] ATLAS collaboration, Search for high-mass dilepton resonances in pp collisions at $\sqrt{s}=8 \mathrm{TeV}$ with the ATLAS detector, Phys. Rev. D 90 (2014) 052005 [arXiv:1405.4123] [INSPIRE].

[52] CMS collaboration, Search for physics beyond the standard model in dilepton mass spectra in proton-proton collisions at $\sqrt{s}=8 \mathrm{TeV}$, JHEP 04 (2015) 025 [arXiv:1412.6302] [INSPIRE].

[53] CMS collaboration, Search for narrow resonances decaying to dijets in proton-proton collisions at $\sqrt{s}=13$ TeV, Phys. Rev. Lett. 116 (2016) 071801 [arXiv:1512.01224] [INSPIRE].

[54] Z. Han and W. Skiba, Effective theory analysis of precision electroweak data, Phys. Rev. D 71 (2005) 075009 [hep-ph/0412166] [INSPIRE].

[55] Z. Han, Electroweak constraints on effective theories with $\mathrm{U}(2) \times(1)$ flavor symmetry, Phys. Rev. D 73 (2006) 015005 [hep-ph/0510125] [INSPIRE].

[56] A. Efrati, A. Falkowski and Y. Soreq, Electroweak constraints on flavorful effective theories, JHEP 07 (2015) 018 [arXiv:1503.07872] [INSPIRE].

[57] CMS collaboration, Search for a Narrow Resonance Produced in 13 TeV pp Collisions Decaying to Electron Pair or Muon Pair Final States, CMS-PAS-EXO-15-005 (2015). 